\title{
THE COMMUTANT OF A CERTAIN COMPRESSION
}

\author{
WILLIAM T. ROSS
}

(Communicated by Palle E. T. Jorgensen)

\begin{abstract}
Let $G$ be any bounded region in the complex plane and $K \subset G$ be a simple compact arc of class $C^{1}$. Let $A^{2}(G \backslash K)$ (resp. $A^{2}(G)$ ) be the Bergman space on $G \backslash K$ (resp. $G$ ). Let $S$ be the operator multiplication by $z$ on $A^{2}(G \backslash K)$ and $C=\left.P_{\mathcal{N}} S\right|_{\mathcal{N}}$ be the compression of $S$ to the semi-invariant subspace $\mathscr{N}=A^{2}(G \backslash K) \ominus A^{2}(G)$. We show that the commutant of $C^{*}$ is the set of all operators of the form $A^{-1} M_{h} A$, where $h$ is a multiplier on a certain Sobolev space of functions on $K$ and $(A f)(w)=\int_{G} f(z)(\bar{z}-\bar{w})^{-1} d A(z)$ $(w \in K)$. We also use multiplier theory in fractional order Sobolev spaces to obtain further information about $C$.
\end{abstract}

\section{INTRODUCTION}

Let $U$ be a bounded region in the complex plane and define $A^{2}(U)$, the Bergman space, to be the Hilbert space of analytic functions on $U$ with $\int_{U}|f(z)|^{2} d A(z)<\infty$. (Here $d A$ is two-dimensional Lebesgue measure.) Define the subnormal multiplication operator $S^{U}$ on $A^{2}(U)$ by

$$
\left(S^{U} f\right)(z)=z f(z) .
$$

It is know [C2] that the commutant of $S^{U}$ (i.e., the set of operators on $A^{2}(U)$ that commute with $S^{U}$ ) is the set of operators of the form $S_{\varphi}^{U}$, where

$$
\left(S_{\varphi}^{U} f\right)(z)=\varphi(z) f(z)
$$

and $\varphi \in H^{\infty}(U)$, the algebra of bounded analytic functions on $U$. We mention that multiplication (and Toeplitz) operators on the Bergman space have been studied in [Ax, ACM] where it is shown that the spectrum $\sigma\left(S_{\varphi}^{U}\right)$ is the closure of $\varphi(U)$ and the essential spectrum $\sigma_{\mathrm{e}}\left(S_{\varphi}^{U}\right)$ is the cluster set of $\varphi$ near the nonremovable singularities of $\partial U$. (Here a point $\lambda \in \partial U$ is removable if there exists a neighborhood $W$ of $\lambda$ such that every $f \in A^{2}(U)$ has an analytic extension to $W \cup U$.)

Let $G$ be a bounded region in the complex plane and $K \subset G$ be a simple compact arc of class $C^{1}$. (Here $C^{1}$ means that $K$ has a continuously differentiable parameterization $\alpha:[a, b] \rightarrow K$ with $\alpha^{\prime}(t) \neq 0$ on $[a, b]$.) The

Received by the editors October 28, 1991; this paper was presented in a special session in Bergman spaces at the 871 st meeting of the American Mathematical Society in Baltimore, MD.

1991 Mathematics Subject Classification. Primary 47B38, 46E15.

Key words and phrases. Bergman spaces, multiplication operators, Sobolev spaces, multipliers. 
Bergman space $A^{2}(G \backslash K)$ can be decomposed as

$$
A^{2}(G \backslash K)=A^{2}(G) \oplus \mathscr{N},
$$

which allows one to decompose $S^{G \backslash K}$ in matricial form as

$$
S^{G \backslash K}=\left(\begin{array}{cc}
S^{G} & B \\
0 & C
\end{array}\right) .
$$

The object of study here is the semi-invariant subspace $\mathscr{N}$ and the compression $C=\left.P_{\mathscr{N}} S^{G \backslash K}\right|_{\mathscr{N}}$ of $S^{G \backslash K}$ to $\mathcal{N}$. In [R1] it was shown that if $G$ is a Jordan domain, then every $f \in \mathcal{N}$ has an analytic continuation across the analytic arcs of $\partial G$, the operators $B$ and $C^{*} C-C C^{*}$ are compact, and $\sigma_{\mathrm{e}}(C)=K$. (Here $\sigma(C)$ is the spectrum and $\sigma_{\mathrm{e}}(C)$ is the essential spectrum of $C$.) We mention that a rough analog for the compression $C$ was studied for the Hardy space $H^{p}(G \backslash K)$ where similar properties were observed, see [C $3, \S \S 4$ and 5].

In [R2] the lattice of invariant subspaces for $C$ was discussed by making the observation that every invariant subspace of $C$ is of the form $\mathscr{M} \ominus A^{2}(G)$, where $\mathscr{M}$ is $S^{G \backslash K}$-invariant and contains $A^{2}(G)$ and then by looking at such $\mathscr{M}$. In the same paper, it was shown how to represent $C^{*}$ as a multiplication operator on a certain Sobolev space of functions on $K$. Define $\mathscr{H}(K)$ as the space of functions in $L^{2}(K,|d z|)(|d z|$ is arc length measure on $K)$ with the following norm finite:

$$
\|h\|_{\mathscr{P}(K)}^{2}=\int_{K}|h(z)|^{2}|d z|+\int_{K} \int_{K} \frac{|h(z)-h(w)|^{2}}{|z-w|^{2}}|d z||d w| .
$$

For $f \in L^{2}(G)$, define the conjugate-Cauchy transform $\tilde{f}$ of $f$ by

$$
\tilde{f}(w)=\int_{G} \frac{f(z)}{\bar{z}-\bar{w}} d A(z) .
$$

In [R2] it was shown that the map $(A f)(w)=\tilde{f}(w)$ is an invertible operator from $\mathscr{N}$ onto $\mathscr{H}(K)$ with $\left(A C^{*} A^{-1} h\right)(w)=\bar{w} h(w)$ for all $h \in \mathscr{H}(K)$. As a matter of fact, if for $\varphi \in H^{\infty}(G)$ we define $C_{\varphi}=\left.P_{\mathscr{N}} S_{\varphi}^{G \backslash K}\right|_{\mathcal{N}}$, then we can prove in a very similar way that $\left(A C_{\varphi}^{*} A^{-1} h\right)(w)=\overline{\varphi(w)} h(w)$ for all $h \in \mathscr{H}(K)$.

The purpose of this paper is to employ this representation of $C^{*}$ in order to compute the commutant of $C$. Since $\mathcal{N}$ is semi-invariant, a theorem of Sarason [S] gives us $C_{\varphi} C_{\psi}=C_{\psi} C_{\varphi}$ for all $\varphi, \psi \in H^{\infty}(G)$. So knowing the commutant of $S^{G \backslash K}$, one might be led to believe that the commutant of $C$ is the set of operators of the form $C_{\varphi}$, where $\varphi \in H^{\infty}(G)$. This, however, is not the case and we state our main theorem, which characterizes the commutant of $C$.

Theorem 1.1. If $Y$ is a continuous operator on $\mathcal{N}$ with $Y C=C Y$, then $Y^{*}=$ $A^{-1} M_{h} A$, where $M_{h} k=h k$ and $h$ is a multiplier on the Sobolev space $\mathscr{H}(K)$.

The fact that $C^{*}$ can be represented as a multiplication operator and that $C^{*} C-C C^{*}$ is compact leads one to question whether or not $C$ is in fact a normal operator, or even similar to a normal operator. Using multiplier theory, as in work by Maz'ya and Shaposhnikova, we answer these two questions in the negative by the following theorem:

Theorem 1.2. The compression $C$ is not similar to a normal operator. 


\section{Preliminaries}

For a bounded region $U$ in the complex plane, let $C_{0}^{\infty}(U)$ denote the set of infinitely differentiable functions with compact support in $U$. Define the Sobolev space $W_{1}^{2,0}(U)$ as the completion of $C_{0}^{\infty}(U)$ in the norm

$$
\|u\|_{W_{1}^{2,0}}=\left(\int_{U}|\nabla u|^{2} d A(z)\right)^{1 / 2} .
$$

Let $B^{2}(U)=L^{2}(U) \ominus A^{2}(U)$ and note that by a result of [AFV] (also see [R2]), $B^{2}(U)$ is the $L^{2}(U)$-closure of $D^{U} C_{0}^{\infty}(U)$, where

$$
D^{U}=\frac{\partial}{\partial z}=\frac{1}{2}\left(\frac{\partial}{\partial x}-i \frac{\partial}{\partial y}\right),
$$

and that $D^{U}$ extends to be a bounded invertible operator from $W_{1}^{2,0}(U)$ onto $B^{2}(U)$. It turns out [R2] that $T^{U}=\left(D^{U}\right)^{-1}$ can be given by $\left(T^{U} g\right)(w)=$ $-\pi^{-1} \tilde{g}(w)$. by

Since $\langle z f, g\rangle=\langle f, \bar{z} g\rangle$ for all $f, g \in L^{2}(U)$, we can define $R^{U}$ on $B^{2}(U)$

$$
\left(R^{U} g\right)(w)=\bar{w} g(w) .
$$

We can also define $M^{U}$ on $W_{1}^{2,0}(U)$ by

$$
\left(M^{U} h\right)(w)=\bar{w} h(w)
$$

and see by [AFV] (see also [R2]) that $D^{U} M^{U}=R^{U} D^{U}$.

For our bounded region $G$ and $K \subset G$, a simple compact arc of class $C^{1}$, the differential operator $D^{G}$ will induce the obvious isomorphism

$$
\widetilde{D^{G}}: W_{1}^{2,0}(G) / W_{1}^{2,0}(G \backslash K) \rightarrow B^{2}(G) / B^{2}(G \backslash K)
$$

by $\widetilde{D^{G}}[h]=\left[D^{G} h\right]$. (Here and throughout the rest of this paper, we let $[j]$ denote the coset of the element $j$.) $R^{G}$ will induce a continuous operator $\widetilde{R^{G}}$ on $B^{2}(G) / B^{2}(G \backslash K)$ by $\widetilde{R^{G}}[g]=\left[R^{G} g\right]$ and $M^{G}$ will induce $\widetilde{M^{G}}$ on $W_{1}^{2,0}(G) / W_{1}^{2,0}(G \backslash K)$ in a similar fashion to get

$$
\widetilde{D^{G}} \widetilde{M^{G}}=\widetilde{R^{G}} \widetilde{D^{G}} .
$$

Next, we define $X: W_{1}^{2,0}(G) \rightarrow \mathscr{H}(K)$ by $X h=\operatorname{tr}(h)$, where $\operatorname{tr}(h)$ is the trace of $h$ on $K$ as defined in [Liz] (also see [MS, Chapter 5]). (Roughly speaking, the trace is the 'restriction' of $h$ to $K$. For example, if $K=[a, b]$, an interval in the real line, we have $\operatorname{tr}(h)(x)=\lim _{y \rightarrow 0} h(x, y)$ a.e. [dx].) $X$ is a continuous surjective operator with $\operatorname{ker}(X)=W_{1}^{2,0}(G \backslash K)$ (see [Liz, R2]), which will induce the continuous invertible operator

$$
\widetilde{X}: W_{1}^{2,0}(G) / W_{1}^{2,0}(G \backslash K) \rightarrow \mathscr{H}(K)
$$

by $\tilde{X}[h]=\operatorname{tr}(h)$.

Note that $\mathscr{N}=B^{2}(G) \ominus B^{2}(G \backslash K)$, so letting $i: \mathscr{N} \rightarrow B^{2}(G) / B^{2}(G \backslash K)$ be the natural map $i(f)=[f]$ we form $A: \mathscr{N} \rightarrow \mathscr{H}(K)$ by

$$
A=-\pi \widetilde{X}\left(\widetilde{D^{G}}\right)^{-1} i
$$

and see [R2] that $(A f)(w)=\tilde{f}(w)$ and $\left(A C^{*} A^{-1} h\right)(w)=\bar{w} h(w)$ for all $h \in$ $\mathscr{H}(K)$. 


\section{Multipliers}

For $\mathscr{B}=W_{1}^{2,0}(G)$ or $\mathscr{B}=\mathscr{H}(K)$ we define the space of multipliers on $\mathscr{B}$. We refer the reader to [MS] for a thorough reference on the subject of multipliers on Sobolev spaces.

A function $h \in \mathscr{B}$ is a multiplier if $g h \in \mathscr{B}$ for all $g \in \mathscr{B}$. An application of the closed graph theorem gives us that if $h$ is a multiplier, then the operator $M_{h}$ on $\mathscr{B}$ defined by $M_{h} g=h g$ is continuous. We denote the space of multipliers by $M \mathscr{B}$ and endow $M \mathscr{B}$ with the multiplier norm

$$
\|h\|_{M \mathscr{B}}=\sup _{\|g\|_{\mathscr{B}} \leq 1}\|g h\|_{\mathscr{B}} .
$$

If $H \in M W_{1}^{2,0}(G)$ then $\operatorname{tr}(H) \in M \mathscr{H}(K)$, and if $h \in M \mathscr{H}(K)$ then there exists an $H \in M W_{1}^{2,0}(G)$ with $\operatorname{tr}(H)=h$. We refer the reader to [MS, Chapter 5; Str] for a discussion of these results.

This next lemma gives us an estimate for the norm of a multiplier in $\mathscr{H}(K)$, which will be used later. A proof can also be found in [MS, Chapter 2].

Lemma 3.1. If $h \in M \mathscr{H}(K)$, then

$\|h\|_{M \mathscr{H}(K)} \sim \sup _{F \text { compact }}\left(\frac{1}{\operatorname{Cap}(F)}\right)^{1 / 2}\left(\int_{F} \int_{K} \frac{|h(z)-h(w)|^{2}}{|z-w|^{2}}|d z||d w|\right)^{1 / 2}+\|h\|_{\infty}$.

Here $\|\cdot\| \sim B$ means that there is a positive constant $c$ with $c^{-1} B \leq\|\cdot\| \leq$ $c B$, and $\operatorname{Cap}(F)$ denotes the logarithmic capacity of $F$.

\section{Proof of Theorem 1.1}

We now proceed to the proof of Theorem 1.1. Let $K$ be parameterized by $\alpha(t), a \leq t \leq b$. We first show that polynomials in the independent variable $z$ are dense in $\mathscr{H}(K)$. The proof is quite simple, but we include it for the sake of completeness.

Lemma 4.1. Let $\psi \in C_{0}^{\infty}(G)$ and define $g(t)=\psi(\alpha(t))$. Then given $\varepsilon>0$, there exists a polynomial $p(z)$ with

$$
\sup _{a \leq t \leq b}|p(\alpha(t))-g(t)|<\varepsilon
$$

and

$$
\sup _{a \leq t \leq b}\left|\frac{d}{d t}(p(\alpha(t))-g(t))\right|<\varepsilon .
$$

Proof. Since $G \backslash K$ is connected, then given $\varepsilon>0$, we apply Lavrientiev's theorem [C2] to find a polynomial $q(z)$ with

$$
\sup _{z \in K}\left|q(z)-\frac{g^{\prime}\left(\alpha^{-1}(z)\right)}{\alpha^{\prime}\left(\alpha^{-1}(z)\right)}\right|<\varepsilon .
$$

Let $p(z)$ be a polynomial with $p^{\prime}=q$ and $p(\alpha(a))=0$. Then

$$
\frac{d}{d t} p(\alpha(t))=q(\alpha(t)) \alpha^{\prime}(t)
$$


and

$$
\begin{aligned}
\left|\frac{d}{d t}(p(\alpha(t))-g(t))\right| & =\left|q(\alpha(t)) \alpha^{\prime}(t)-g^{\prime}(t)\right| \\
& =\left|\alpha^{\prime}(t)\right|\left|q(\alpha(t))-\frac{g^{\prime}(t)}{\alpha^{\prime}(t)}\right| \leq M \varepsilon,
\end{aligned}
$$

where $M=\sup \left\{\left|\alpha^{\prime}(t)\right|: a \leq t \leq b\right\}$. Thus

$$
\sup _{a \leq t \leq b}|p(\alpha(t))-g(t)|=\sup _{a \leq t \leq b}\left|\int_{a}^{t}\left(\frac{d}{d s} p(\alpha(s))-g^{\prime}(s)\right) d s\right| \leq(b-a) M \varepsilon \text {. }
$$

Lemma 4.2. Polynomials in $z$ are dense in $\mathscr{H}(K)$.

Proof. Since the trace operator $X$ is continuous, $\left.C_{0}^{\infty}(G)\right|_{K}$ is dense in $\mathscr{H}(K)$. So let $\psi \in C_{0}^{\infty}(G)$ and $\varepsilon>0$ be given. By Lemma 4.1, there is a polynomial $p(z)$ with

$$
\sup _{a \leq t \leq b}|p(\alpha(t))-\psi(\alpha(t))| \leq \varepsilon
$$

and

$$
\sup _{a \leq t \leq b}\left|\frac{d}{d t}(p(\alpha(t))-\psi(\alpha(t)))\right| \leq \varepsilon .
$$

Let $h(z)=p(z)-\psi(z)$. Then

$$
\begin{aligned}
\|h\|_{\mathscr{H}(K)}^{2}= & \int_{K}|h(z)|^{2}|d z|+\int_{K} \int_{K} \frac{|h(z)-h(w)|^{2}}{|z-w|^{2}}|d z||d w| \\
= & \int_{a}^{b}|h(\alpha(t))|^{2}\left|\alpha^{\prime}(t)\right|^{2} d t \\
& +\int_{a}^{b} \int_{a}^{b} \frac{|h(\alpha(t))-h(\alpha(s))|^{2}}{|\alpha(t)-\alpha(s)|^{2}}\left|\alpha^{\prime}(t)\right|\left|\alpha^{\prime}(s)\right| d s d t \\
\leq & C\left(\int_{a}^{b}|h(\alpha(t))|^{2} d t+\int_{a}^{b} \int_{a}^{b} \frac{|h(\alpha(t))-h(\alpha(s))|^{2}}{|s-t|^{2}} d s d t\right) \leq C^{\prime} \varepsilon .
\end{aligned}
$$

We now prove Theorem 1.1.

Proof. Let $Y$ be a continuous operator on $\mathscr{N}$ with $Y C=C Y$. Then $Y^{*} C^{*}=$ $C^{*} Y^{*}$, so $B=A Y^{*} A^{-1}$ commutes with multiplication by $\bar{z}$ on $\mathscr{H}(K)$.

Let $h=B(1)$ and note that $B(\bar{p})=B(\bar{p} \cdot 1)=\bar{p} h$ for all polynomials $p(z)$. If $f \in \mathscr{H}(K)$, choose a sequence of polynomials $p_{n}$ with $\bar{p}_{n} \rightarrow f$ in $\mathscr{H}(K)$. We assume (by passing to a subsequence if necessary) that $\bar{p}_{n} \rightarrow f$ a.e. on $K$. Now $B\left(\bar{p}_{n}\right) \rightarrow B(f)$ in $\mathscr{H}(K)$ and $B\left(\bar{p}_{n}\right)=h \bar{p}_{n}$ converges to $h f$ a.e. on $K$, so $B(f)=h f$ a.e., making $h$ a multiplier on $\mathscr{H}(K)$.

From this theorem and some multiplier theory, we can now express the commutant of $C$ in terms of multipliers on $W_{1}^{2,0}(G)$.

Corollary 4.1. Let $Y$ be a continuous operator on $\mathscr{N}$ with $Y C=C Y$. Then

$$
Y^{*}=\left.P_{\mathscr{N}} D^{G} M_{H}\left(D^{G}\right)^{-1}\right|_{\mathscr{N}}
$$

for some $H \in M W_{1}^{2,0}(G)$.

Proof. By Theorem 1.1, $Y^{*}=A^{-1} M_{h} A$ for some $h \in M \mathscr{H}(K)$. So

$$
Y^{*}=i^{-1} \widetilde{D}^{G} \tilde{X}^{-1} M_{h} \tilde{X} \widetilde{D}^{G^{-1}} i \text {. }
$$


Letting $H \in M W_{1}^{2,0}(G)$ with $\operatorname{tr}(H)=h$ we get that for any $f \in \mathscr{N}$

$$
\begin{aligned}
Y^{*} f & =i^{-1} \widetilde{D}^{G} \widetilde{X}^{-1}(h \tilde{f})=i^{-1} \widetilde{D}^{G}[H \tilde{f}] \\
& =i^{-1}\left[D^{G} M_{H}\left(D^{G}\right)^{-1} f\right]=P_{\mathscr{N}}\left(D^{G}\right)^{-1} f .
\end{aligned}
$$

We also point out that as a consequence of Theorem 1.1, not every operator in the commutant of $C$ is of the form $C_{\psi}$ for some $\psi \in H^{\infty}(G)$. Let $h$ be an infinitely differentiable function on $K$ with no co-analytic extension to $G$. Then if $B=A^{-1} M_{h} A, B^{*}$ commutes with $C$. If $B^{*}=C_{\psi}$ for some $\psi \in H^{\infty}(G)$, then using the fact that $A C_{\psi}^{*} A^{-1}=M_{\bar{\psi}}$ on $\mathscr{H}(K)$, we have $h=\bar{\psi}$ on $K$, which is a contradiction.

\section{5. $C$ IS NOT SIMILAR TO A NORMAL OPERATOR}

The operator $C^{*}$ can be represented as a multiplication operator on $\mathscr{H}(K)$, and $C^{*} C-C C^{*}$ is compact, which brings up the question of the normality of $C$. We will now employ Lemma 3.1 and the spectral theorem for normal operators to prove that $C$ is not similar to a normal operator.

Proof of Theorem 1.2. We prove this for $K=[a, b]$ an interval in the real line and note that the proof for the general $K$ is done in a similar manner.

If $C$, equivalently $C^{*}$, were similar to a normal operator, then by the above comments, $M_{x}$ (multiplication by $x$ ) on $\mathscr{H}([a, b]$ ) would be similar to a normal operator. Without loss of generality, we can assume that $[a, b]=$ $[-1,1]$. If $M_{x}$ were similar to a normal operator, then by the spectral theorem for normal operators, we would have that for all $\mu \notin[-1,1]$,

$$
\left\|\left(M_{x-\mu}\right)^{-1}\right\| \leq C(\operatorname{dist}(\mu,[-1,1]))^{-1}
$$

for some $C>0$ independent of $\mu$. Let $\mu=i \lambda, 0<\lambda<1$. We will show that

$$
\sup _{0<\lambda<1} \operatorname{dist}(\mu,[-1,1])\left\|\left(M_{x-i \lambda}\right)^{-1}\right\|=+\infty,
$$

which will rule out the normality (or similar to a normal) of $M_{x}$ and hence $C$. By Lemma 3.1, we know that

$$
\begin{aligned}
\left\|\left(M_{x-i \lambda}\right)^{-1}\right\| \geq & C \sup _{E \text { compact }}\left(\frac{1}{\operatorname{Cap}(E)}\right)^{1 / 2} \\
& \times\left(\int_{E} \int_{E} \frac{|1 /(x-i \lambda)-1 /(y-i \lambda)|^{2}}{|x-y|^{2}} d x d y\right)^{1 / 2}+\left\|\frac{1}{x-i \lambda}\right\|_{\infty} \\
\text { (2) } \quad \geq & C \sup _{0<r<1}\left(\frac{1}{\operatorname{Cap}([-r, r])}\right)^{1 / 2}\left(\int_{-r}^{r} \int_{-r}^{r} \frac{d x d y}{|x-i \lambda|^{2}|y-i \lambda|^{2}}\right)^{1 / 2}+\frac{1}{\lambda} .
\end{aligned}
$$

An estimate on logarithmic capacity (see [MS, Chapter 2]), yields

$$
\operatorname{Cap}([-r, r]) \sim\left(\log \left(\frac{2}{r}\right)\right)^{-1}
$$

for all $0<r<1$. Using this will give us that equation (3) is bounded below by

$$
C^{\prime} \sup _{0<r<1}\left(\log \left(\frac{2}{r}\right)\right)^{1 / 2} \int_{-r}^{r} \frac{d x}{|x-i \lambda|^{2}}+\frac{1}{\lambda} \text {. }
$$


Since $\operatorname{dist}(i \lambda,[-1,1])=\lambda$, we can prove equation (1) by showing that

$$
\sup _{0<\lambda<1} \sup _{0<r<1} \lambda\left(\log \left(\frac{2}{r}\right)\right)^{1 / 2} \int_{-r}^{r} \frac{d x}{|x-i \lambda|^{2}}=+\infty .
$$

Note that

$$
\int_{-r}^{r} \frac{d x}{|x-i \lambda|^{2}} \geq \frac{2 r}{|r-i \lambda|^{2}}=\frac{2 r}{r^{2}+\lambda^{2}}
$$

so the left-hand side of equation (5) is bounded below by

$$
\sup _{0<\lambda<1} \sup _{0<r<1} \lambda\left(\log \left(\frac{2}{r}\right)\right)^{1 / 2} \frac{2 r}{r^{2}+\lambda^{2}} .
$$

Letting $r=\lambda$ we get that equation (6) is bounded below by

$$
\sup _{0<r<1}\left(\log \left(\frac{2}{r}\right)\right)^{1 / 2}=+\infty .
$$

Hence $M_{x}$, and thus $C$, is not similar to a normal operator.

\section{REFERENCES}

[Ax] S. Axler, Multiplication operators on Bergman spaces, J. Reine Angew. Math. 336 (1982), 26-44.

[AAS] D. R. Adams, N. Aronszajn, and K. T. Smith, Theory of Bessel potentials (Part II), Ann. Inst. Fourier (Grenoble) 17 (1962), 1-135.

[ACM] S. Axler, J. B. Conway, and G. McDonald, Toeplitz operators on Bergman spaces, Canad. J. Math. 34 (1982), 466-483.

[AFV] C. Apostol, C. Fioaş, and D. Voiculescu, Some results on non-quasitriangular operators. IV, Rev. Roumaine Math. Pures Appl. 18 (1973).

[AS] N. Aronszajn and K. T. Smith, Theory of Bessel potentials (Part I), Ann. Inst. Fourier (Grenoble) 11 (1961), 385-475.

[C1] J. B. Conway, The dual of a subnormal operator, J. Operator Theory 5 (1981), 195-211.

[C2] _ _ Subnormal operators, Pitman Press, Boston, MA, 1981.

[C3] _ - Spectral properties of certain operators on Hardy spaces of planar domains, Integral Equations Operator Theory 10 (1987), 659-706.

[Liz] P. I. Lizorkin, Boundary properties of functions from 'weighted' classes, Dokl. Akad. Nauk SSSR 132 (1960), 514-517; English transl., Soviet Math. Dokl. 1 (1960), 589-593.

[MS] V. G. Maz'ya and T. O. Shaposhnikova, Theory of multipliers in spaces of differentiable functions, Pitman Press, Boston, MA, 1985.

[R1] W. T. Ross, Analytic continuation in Bergman spaces and the compression of certain Toeplitz operators, Indiana Univ. Math. J. 40 (1991), 1363-1386.

[R2] _ Multiplication operators on Bergman spaces and invariant subspaces, thesis, Univ. of Virgina, 1991.

[S] D. Sarason, On spectral sets having connected complement, Acta Sci. Math. Szeged 26 (1965), 261-266.

[Str] R. Strichartz, Multipliers on fractional order Sobolev spaces, J. Math. Mech. 16 (1967), 1031-1060.

Department of Mathematics, Lafayette College, Easton, Pennsylvania 18042

Current address: Department of Mathematics, University of Richmond, Richmond, Virginia 23173

E-mail address: rossb@ aurora.urich.edu 